\title{
Parallel Scan-Like Test and Multiple-Defect Diagnosis for Digital Microfluidic Biochips
}

\author{
Tao Xu, Student Member, IEEE, and Krishnendu Chakrabarty, Member, IEEE
}

\begin{abstract}
Dependability is an important attribute for microfluidic biochips that are used for safety-critical applications such as point-of-care health assessment, air-quality monitoring, and food-safety testing. Therefore, these devices must be adequately tested after manufacture and during bioassay operations. We propose a parallel scan-like testing methodology for digital microfluidic devices. A diagnosis method based on test outcomes is also proposed. The diagnosis technique is enhanced such that multiple defect sites can be efficiently located using parallel scan-like testing. Defect diagnosis can be used to reconfigure a digital microfluidic biochip such that faults can be avoided, thereby enhancing chip yield and defect tolerance. We evaluate the proposed method using complexity analysis as well as applying it to a fabricated biochip.
\end{abstract}

Index Terms-Digital microfluidics, fault detection, fault diagnosis, lab-on-chip.

\section{INTRODUCTION}

$\mathbf{R}$ ECENT advances in microfluidics technology have led to the emergence of miniaturized biochip devices for biochemical analysis [1]-[6]. Microfluidics-based biochips, also referred to as lab-on-a-chip, are replacing cumbersome and expensive laboratory equipment for applications such as high-throughput sequencing, parallel immunoassays, protein crystallization, blood chemistry for clinical diagnostics, and environmental toxicity monitoring. Biochips offer the advantages of higher sensitivity, lower cost due to smaller sample and reagent volumes, higher levels of system integration, and less likelihood of human error.

Currently, most commercially available biochips are based on continuous fluid permanently etched microchannels [2], [7], [8]. Fluid flow in these devices is controlled either using micropumps and microvalves, or by electrical methods based on electrokinetics and electroosmosis [2]. FlowFETs, where electroosmostic fluid flow is controlled by a gate electrode similar to the behavior of a MOSFET, have also been proposed in [7].

An alternative category of microfluidic biochips, referred to as "digital microfluidics" relies on the principle of electrowetting-on-dielectric [1]. Bioassay protocols are scaled down (in

Manuscript received May 15, 2007; revised August 6, 2007. This work was supported in part by the National Science Foundation under Grant IIS-0312352 and Grant CCF-0541055. A preliminary version of this paper was published in the Proceedings of the 2007 IEEE European Test Conference, pp. 63-68. This paper was recommended by Associate Editor B.-D. Liu.

The authors are with the Department of Electrical and Computer Engineering, Duke University, Durham, NC 27708 USA (e-mail: tx@ee.duke.edu; krish@ee. duke.edu).

Color versions of one or more of the figures in this paper are available online at http://ieeexplore.ieee.org.

Digital Object Identifier 10.1109/TBCAS.2007.909025 terms of liquid volumes and assay times), and run on a microfluidic chip by manipulating discrete droplets of nanoliter volume using a patterned array of electrodes. By reducing the rate of sample and reagent consumption, digital microfluidic biochips enable continuous sampling and analysis for online, real-time, chemical and biological analysis. These systems also have dynamic reconfigurability, whereby microfluidic modules can be relocated to other places on the electrode array, without affecting the functionality, during the concurrent execution of a set of bioassays [7]. Reconfigurability enables the design of multifunctional and "smart" microfluidic biochips that can be used for a wide variety of applications. Moreover, defects can be tolerated through system reconfiguration after testing and fault diagnosis.

As chemists and biologists map more bioassays on a microfluidic platform for concurrent execution, system complexity and integration levels are expected to increase steadily. However, as in the case of integrated circuits, an increase in density and area of microfluidics-based biochips will reduce yield, especially for newer technologies. Moreover, to reduce cost for disposable devices, device manufacturers are investigating inexpensive processes and materials for low-cost biochip fabrication [9]. As a result, microfluidic biochips are likely to suffer from high defect densities.

Dependability is an important system attribute for biochips that are used for safety-critical applications such as point-of care diagnostics, health assessment and screening for infectious diseases, air-quality monitoring, and food-safety tests, as well as for pharmacological procedures for drug design and discovery that require high precision levels. Some manufacturing defects may be latent, and they may produce errors during field operation. In addition, harsh operational environments and biological samples (e.g., proteins) may introduce physical defects such as particle contamination and residue on surfaces due to adsorption. Therefore, biochip platforms must be adequately tested after manufacture, before the start of a bioassay, and during bioassay execution. Moreover, since disposable biochips are being targeted for a highly competitive and low-cost market segment, test and diagnosis methods must be inexpensive, quick and effective.

We propose a cost-effective testing methodology referred to as "parallel scan-like test" for droplet-based microfluidic devices [10], as well as a rapid multiple defects diagnosis method based on test outcomes. The proposed method allows testing using parallel droplet pathways in both online and offline test scenarios. The diagnosis outcome can be used to reconfigure a droplet-based biochip such that faults can be easily avoided. In this way, the yield for these devices can be increased (through re- 


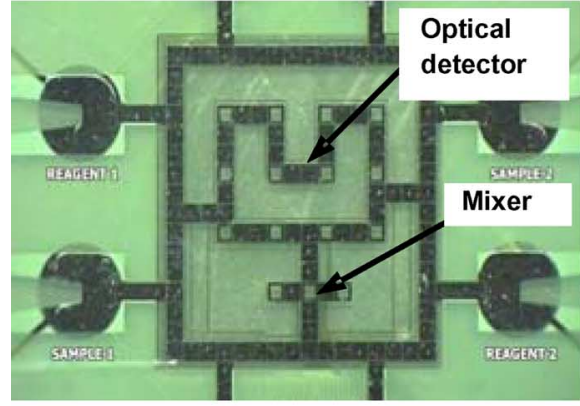

Glass-substrate platform

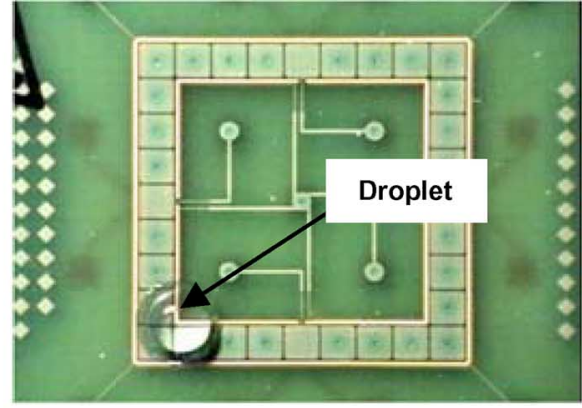

PCB platform

Fig. 1. Fabricated digital microfluidic arrays.

configuration after manufacture test and diagnosis), and in-field defect tolerance can be achieved. We evaluate the test and diagnosis methods using complexity analysis as well as experiments using a fabricated biochip.

The rest of the paper is organized as follows. Section II provides an overview of digital microfluidic biochips. In Section III, we discuss related prior work on defect modeling, fault detection, and diagnosis. In Section IV, we first relate defects in microfluidic biochips to fault models and observable errors. We next introduce the proposed parallel "scan-like" test and defect diagnosis scheme for both online and offline testing. Physical defects for microfluidic biochips are listed and fault models are presented. In Section V, we describe an efficient diagnosis scheme that can easily detect and diagnose multiple defects. Next in Section VI, we determine the complexity of the test and diagnosis procedures in terms of the droplet manipulation steps required, and apply these techniques to a fabricated chip in Section VII. Finally, conclusions are drawn in Section VIII.

\section{Digital Microfluidic Biochips}

A digital microfluidic biochip utilizes the phenomenon of electrowetting to manipulate and move microliter or nanoliter droplets containing biological samples on a two-dimensional electrode array [1]. A unit cell in the array includes a pair of electrodes that acts as two parallel plates. The bottom plate contains a patterned array of individually controlled electrodes, and the top plate is coated with a continuous ground electrode. A droplet rests on a hydrophobic surface over an electrode, as shown in Fig. 1. It is moved by applying a control voltage to an electrode adjacent to the droplet and, at the same time, deactivating the electrode just under the droplet. This electronic method of wettability control creates interfacial tension gradients that move the droplets to the charged electrode. Using the electrowetting phenomenon, droplets can be moved to any location on a two-dimensional array.

By varying the patterns of control voltage activation, many fluid-handling operations such as droplet merging, splitting, mixing, and dispensing can be executed in a similar manner. For example, mixing can be performed by routing two droplets to the same location and then turning them about some pivot points. The digital microfluidic platform offers the additional advantage of flexibility, referred to as reconfigurability, since fluidic operations can be performed anywhere on the array.
Droplet routes and operation scheduling result are programmed into a microcontroller that drives electrodes in the array. In addition to electrodes, optical detectors such as LEDs and photodiodes are also integrated in digital microfluidic arrays to monitor colorimetric bioassays [3].

\section{RELATED PRIOR WORK}

An excellent overview of prior work on the testing of microfluidic biochips can be found in [11]. Techniques for fault modeling and fault simulation for continuous-flow microfluidic biochips have been proposed in [12] and [13].

For digital microfluidic chips, techniques for defect classification, test planning, and test resource optimization have recently been presented. A defect classification and test application procedure are described in [14] and [15]. Defects have been classified as being either catastrophic or parametric, and techniques have been developed to detect these defects by electrostatically controlling and tracking droplet motion. The work in [14] and [15] facilitates concurrent testing, which allows fault detection and biomedical assays to run simultaneously on a microfluidic system [16]. These test methods add fluid handling aspects to MEMS testing techniques [17]. A drawback of [14], however, is that it does not present any automated techniques for optimizing the test application procedure. A test planning and test resource optimization method is described in [17]. The test planning problem is mapped to the Hamilton cycle problem from graph theory, which is known to be NP-complete [18]. A heuristic algorithm is therefore proposed to solve the problem. However, this method is based on Monte Carlo simulation and the derivation of the test plans takes a large amount of computation time. In adaptive test environments, where test application must be altered in response to fault detection, this approach is not feasible. Moreover, this method leads to a test plan that is inflexible because it is specific to a target biochip with a fixed array size, and it does not address the problem of fault of diagnosis.

An alternative method for testing digital microfluidic biochips is based on Euler paths [18]. This method maps a digital microfluidic biochip to an undirected graph and an Euler path is determined for it. A test droplet is dispensed from a source reservoir and routed along the derived Euler path, and it passes through all the cells in the array. The test droplet is then routed to the sink reservoir. A probe cell is positioned at the electrode in front of the sink reservoir to analyze the test 
TABLE I

List OF CATASTROPHIC DEFECTS FOR BIOCHIPS

\begin{tabular}{|c|c|c|c|c|}
\hline $\begin{array}{l}\text { Cause of } \\
\text { defect }\end{array}$ & $\begin{array}{l}\text { Defect } \\
\text { type }\end{array}$ & $*$ & $\begin{array}{l}\text { Fault } \\
\text { model }\end{array}$ & $\begin{array}{l}\text { Observable } \\
\text { error }\end{array}$ \\
\hline $\begin{array}{l}\text { Excessive } \\
\text { actuation } \\
\text { voltage } \\
\text { applied to an } \\
\text { electrode }\end{array}$ & $\begin{array}{l}\text { Dielectric } \\
\text { breakdown }\end{array}$ & 1 & $\begin{array}{l}\text { Droplet-electrode } \\
\text { short (a short } \\
\text { between the } \\
\text { droplet and the } \\
\text { electrode) }\end{array}$ & $\begin{array}{l}\text { Droplet undergoes } \\
\text { electrolysis, which } \\
\text { prevents its further } \\
\text { transportation }\end{array}$ \\
\hline $\begin{array}{l}\text { Electrode } \\
\text { actuation for } \\
\text { excessive } \\
\text { duration } \\
\end{array}$ & $\begin{array}{l}\text { Irreversible } \\
\text { charge } \\
\text { concentration } \\
\text { on an electrode } \\
\end{array}$ & 1 & $\begin{array}{l}\text { Electrode-stuck-on } \\
\text { (the electrode } \\
\text { remains constantly } \\
\text { activated) }\end{array}$ & $\begin{array}{l}\text { Unintentional } \\
\text { droplet } \\
\text { operations or stuck } \\
\text { droplets }\end{array}$ \\
\hline $\begin{array}{l}\text { Excessive } \\
\text { mechanical } \\
\text { force applied to } \\
\text { the chip }\end{array}$ & $\begin{array}{l}\text { Misalignment } \\
\text { of parallel } \\
\text { plates } \\
\text { (electrodes and } \\
\text { ground plane) } \\
\end{array}$ & 1 & $\begin{array}{l}\text { Pressure gradient } \\
\text { (net static pressure } \\
\text { in some direction) }\end{array}$ & $\begin{array}{l}\text { Droplet } \\
\text { transportation } \\
\text { without activation } \\
\text { voltage }\end{array}$ \\
\hline Coating failure & $\begin{array}{l}\text { Non-uniform } \\
\text { dielectric layer }\end{array}$ & 1 & $\begin{array}{l}\text { Dielectric islands } \\
\text { (islands of Teflon } \\
\text { coating) }\end{array}$ & $\begin{array}{l}\text { Fragmentation of } \\
\text { droplets and their } \\
\text { motion is } \\
\text { prevented }\end{array}$ \\
\hline \multirow{3}{*}{$\begin{array}{l}\text { Abnormal } \\
\text { metal layer } \\
\text { deposition } \\
\text { and etch } \\
\text { variation } \\
\text { during } \\
\text { fabrication }\end{array}$} & $\begin{array}{l}\text { Grounding } \\
\text { Failure }\end{array}$ & 1 & $\begin{array}{l}\text { Floating droplets } \\
\text { (droplet are not } \\
\text { anchored) }\end{array}$ & $\begin{array}{l}\text { Failure of droplet } \\
\text { transportation }\end{array}$ \\
\hline & $\begin{array}{l}\text { Broken } \\
\text { wire to control } \\
\text { source }\end{array}$ & 1 & $\begin{array}{l}\text { Electrode open } \\
\text { (electrode } \\
\text { actuation is not } \\
\text { possible) }\end{array}$ & $\begin{array}{l}\text { Failure to } \\
\text { activate the } \\
\text { electrode for } \\
\text { droplet } \\
\text { transportation }\end{array}$ \\
\hline & $\begin{array}{l}\text { Metal } \\
\text { connection } \\
\text { between two } \\
\text { adjacent } \\
\text { electrodes } \\
\end{array}$ & 2 & $\begin{array}{l}\text { Electrode short } \\
\text { (short between } \\
\text { electrodes) }\end{array}$ & \multirow{2}{*}{$\begin{array}{l}\text { A droplet resides } \\
\text { in the middle of } \\
\text { the two shorted } \\
\text { electrodes, and its } \\
\text { transport along } \\
\text { one or more } \\
\text { directions cannot } \\
\text { be achieved }\end{array}$} \\
\hline $\begin{array}{l}\text { Particle } \\
\text { contamination } \\
\text { or liquid } \\
\text { residue }\end{array}$ & $\begin{array}{l}\text { A particle that } \\
\text { connect two } \\
\text { adjacent } \\
\text { electrodes }\end{array}$ & 2 & Electrode short & \\
\hline \multirow{2}{*}{$\begin{array}{l}\text { Protein } \\
\text { absorption } \\
\text { during a } \\
\text { bioassay }\end{array}$} & \multirow[t]{2}{*}{$\begin{array}{l}\text { Sample residue } \\
\text { on electrode } \\
\text { surface }\end{array}$} & \multirow[t]{2}{*}{1} & $\begin{array}{l}\text { Resistive open at } \\
\text { electrode }\end{array}$ & $\begin{array}{l}\text { Droplet } \\
\text { transportation is } \\
\text { impeded. }\end{array}$ \\
\hline & & & Contamination & $\begin{array}{l}\text { Assay results are } \\
\text { outside the range } \\
\text { of possible } \\
\text { outcomes }\end{array}$ \\
\hline
\end{tabular}

* Number of cells involved in the defect.

outcome using capacitive detection. Despite its effectiveness for detecting electrode shorts as explained in [19], testing based on an Euler path suffers from long test application time. This approach uses only one droplet to traverse the complete microfluidic array, irrespectively of the array size. Fault diagnosis is carried out by using multiple test application steps and adaptive Euler paths. Such a diagnosis method is inefficient since defect-free cells are tested multiple times. Moreover, as in [17], the method proposed in [19] also leads to a test plan that is specific to a target biochip. If the array dimensions are changed, the test plan must be completely altered. Moreover, to facilitate chip testing in the field, test plans need to be programmed into a microcontroller. However, the hardware implementations of test plans from [17] and [19] are prohibitively expensive, especially for low cost, disposable biochips. Therefore, there is a need for test and diagnosis methods that are efficient and amenable to low-cost hardware implementation of test procedures.

\section{Parallel Scan-Like Test Methodology}

In this section, we present efficient fault detection and diagnosis methods based on multiple-droplet testing. The key idea here is to manipulate multiple droplets in parallel to test the microfluidic array in a scan-like manner.

Table I lists common defects and their causes. It also maps each defect to a fault model and an observable error. Note that all of these defects are catastrophic, i.e., their occurrence will cause the chip to fail. Compared to the defects listed in [14], we have identified several new defects that either result from manufacturing problems or appear during bioassay execution, e.g., electrode charging, misaligned parallel plates, and grounding failure. Note that even though the causes for these new defects are unique, their observable errors are already included in the set of errors targeted in [14]. Therefore, all the defects listed in Table I can be detected by manipulating test droplets to traverse the candidate faulty electrodes. Note that to detect an electrode-short defect, a test droplet needs to traverse two adjacent 


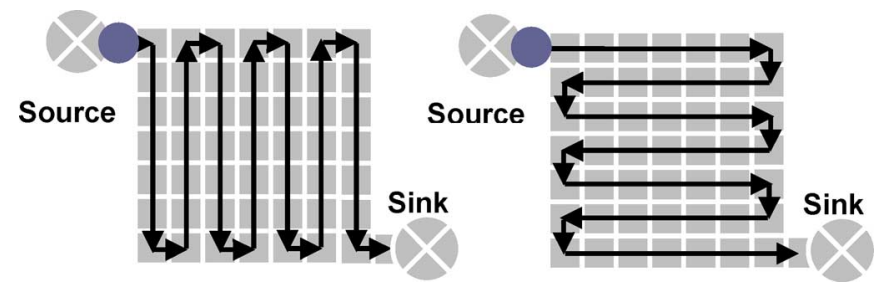

Fig. 2. Illustration of single droplet scan-like test using a single droplet.

electrodes that are involved in the short. The test droplet will reside in the middle of the two shorted electrodes that are activated simultaneously; there will not be sufficient overlap area with the next electrode for further transportation.

Most prototype digital microfluidic devices consist of a twodimensional array of electrodes with one or more sources and sinks on the boundary, as shown in Fig. 2 [9]. In this regular structure, electrodes are carefully aligned in columns and rows. We next describe the parallel scan-like test method, named thus because it manipulates multiple test droplets in parallel to traverse the target microfluidic array, just as test stimuli can be loaded in parallel to multiple scan chains in integrated circuits.

We first describe the special case of a single test droplet. We determine the pathway for the test droplet, irrespective of the bioassay operation, as shown in Fig. 2. Starting from the droplet source, the test droplet follows the pathway to traverse every cell in the array, and it finally reaches the sink. During concurrent testing, a test droplet is guided to visit the available cells in accordance with a predetermined path. If the target cell is temporarily unavailable for testing, i.e., it is occupied by a droplet or it is adjacent to active microfluidic modules, the test droplet waits in the current position until the target cell becomes available. The test outcome is read out using a capacitive sensing circuit connected to the electrode for the sink reservoir, as shown in Fig. 3. Then figure shows details about the set setup and how it was validated. This single-droplet, scan-like algorithm is easy to implement. Moreover, the test plan is general, in the sense that it can be applied to any microfluidic array and for various bioassay operations.

However, in this simple test procedure method, $N \times M$ steps (clock cycles for droplet actuation) are needed for the test droplet to traverse an $N \times M$ microfluidic array. As a result, the test time may be excessive for large arrays. For example, a 600000 -electrode-array manufactured by Silicon Biosystems (based on dielectrophrasis) will require 600000 clock cycles [20]. At a typical actuation clock frequency of $1 \mathrm{~Hz}$, this amounts to seven days of test application time! Moreover, in online testing, the test droplet may have to be stalled several times, and each time a long waiting period may be necessary. Finally, the test outcome for the single-droplet scan-like test provides no diagnostic information. If any cell in the array is faulty, the capacitive sensing circuit will have no readout; however, it is not possible to identify the location of the faulty cell.

The above problems can be tackled by carrying out the scanlike test in parallel using multiple droplets. Each column/row in the array is associated with a test droplet and its "target region." A target region for a droplet includes the cells that are traversed by this droplet. The proposed method can be viewed as carrying out a single-droplet scan-like test in different target regions in

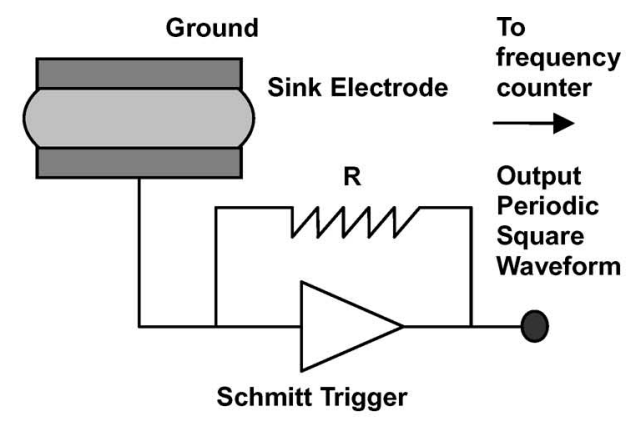

(a)

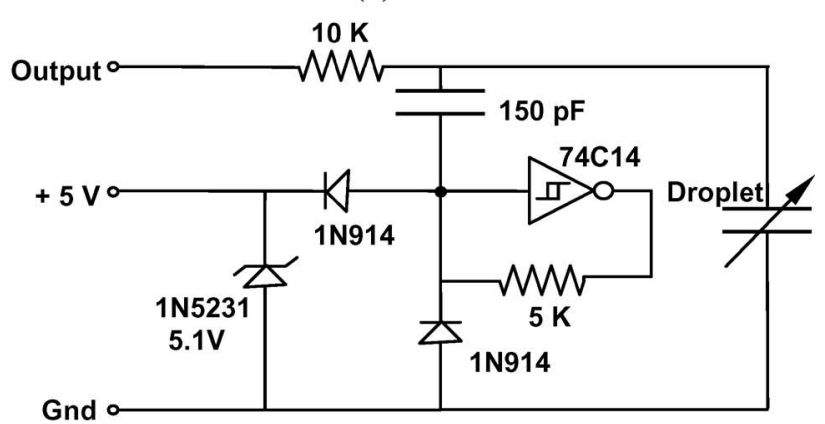

(b)

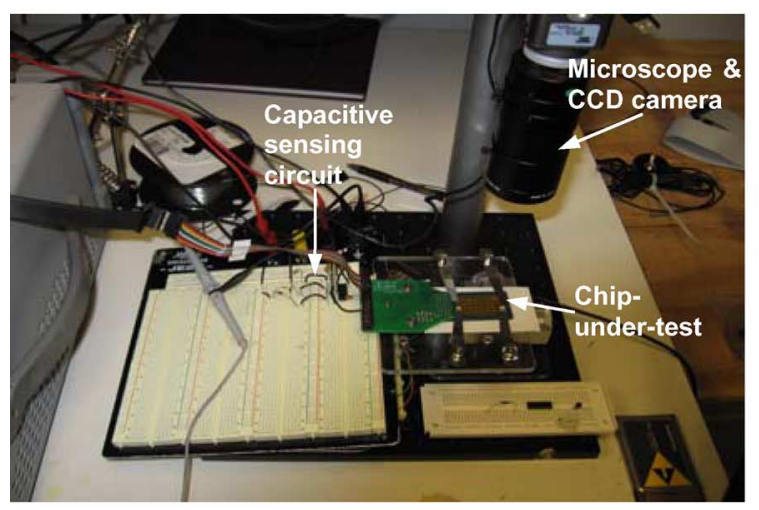

(c)

Fig. 3. Capacitive sensing circuit: (a) outline [11]; (b) detail circuit design; and (c) experimental setup.

parallel, therefore we refer to this method as the parallel scanlike method.

\section{A. Offline Test and Diagnosis}

In offline testing, test droplets are dispensed from the test droplet source to the start electrodes of their target regions. Since we use columns/rows as target regions, the start electrodes are located on the array boundary, as shown in Fig. 4. For each target region, the start electrode acts as the test-droplet source for the underlying single-droplet scan-like method. Therefore, we refer to start electrodes here as pseudo-sources. Starting from these pseudo-sources, test droplets are routed in parallel (similar to a waterfall in nature) to the electrodes at the other end of the corresponding target regions. These end-points are referred to as pseudo-sinks. Finally, the test droplets are routed to the sink reservoir. Note that in above method, we assume that a microfluidic array has only one source and one sink reservoir to facilitate chip packaging and reduce fabrication cost. Dispensed 


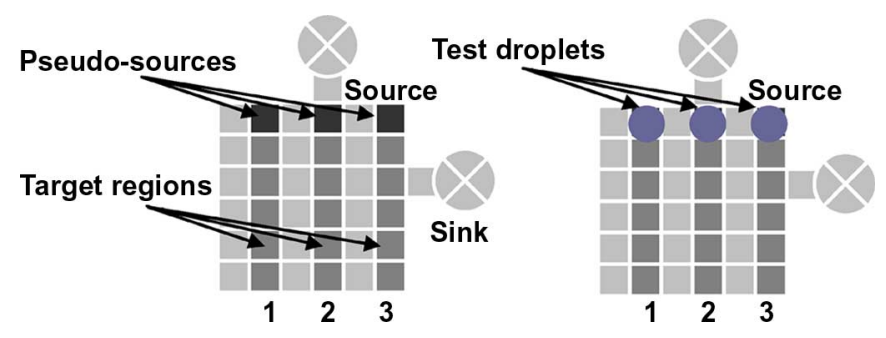

Fig. 4. Example of target regions and pseudo-sources.

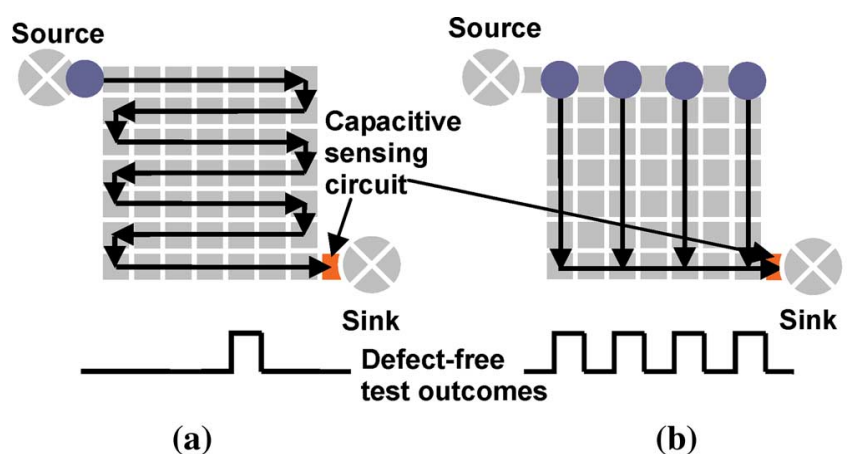

Fig. 5. Example defect-free test outcome for (a) single-droplet scan-like method, and (b) parallel scan-like method.

from the single source, test droplets are aligned one-by-one and routed in sequence, like components in an assembly line, along the periphery nodes to their pseudo-sources. The reverse process is carried out when the test droplets are routed from the pseudo-sink to the sink reservoir.

As in [21], the test outcome is read out using the capacitive sensing circuit located at the sink reservoir. The major enhancement here is that multiple test droplets can be detected at the sink. Instead of a single pulse, the capacitive sensing circuit can detect a pulse sequence corresponding to multiple test droplets; see Fig. 5. Different fault patterns (i.e., groups of failing cells) are mapped to different pulse sequences.

Sufficient spacing between droplets must always be maintained during droplet routing [22]. Therefore, in order to avoid unintentional merging of droplets, test droplets must be at least one electrode away from each other. This implies that only half of the total number of columns/rows can be tested in one parallel scan-like test iteration. Two iterations are needed to detect defects involving single cells in the array. For defects involving two cells, e.g., shorts between two adjacent electrodes, all pairs of adjacent electrode must be tested. Therefore, four iterations are needed to test the microfluidic array-two iterations for the vertically connected pairs and two additional iterations to traverse all the horizontal connections. In addition, a "peripheral test" is carried out before parallel scan-like testing to ensure that a test droplet can be correctly dispensed from the source to pseudo-sources and routed from pseudo-sinks to the sink.

The complete parallel scan-like test procedure is as follows.

Step I. Peripheral Test: A test droplet is dispensed from the source. It is routed to traverse all the peripheral electrodes, and the droplet finally returns to the sink, as shown in Fig. 6.

Step II. Column Test: Two iterations of parallel scan-like test with one column shift are carried out. This step tests

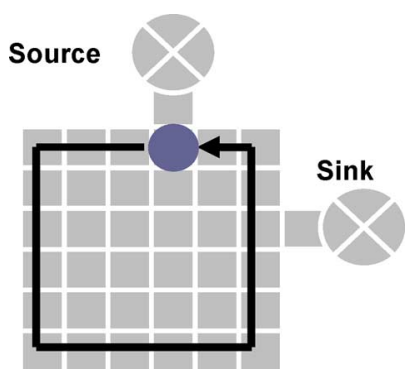

Fig. 6. Step I (peripheral testing).

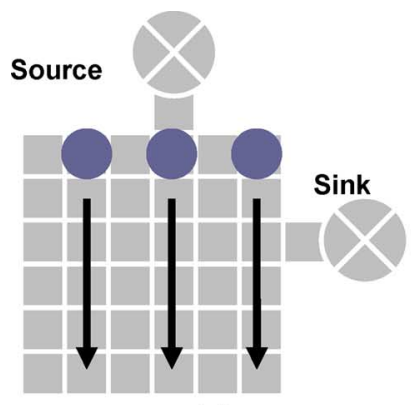

(a)

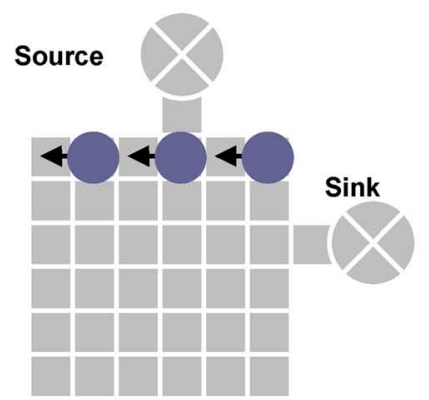

(c)

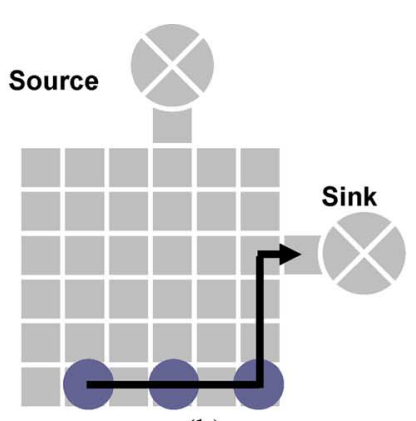

(b)

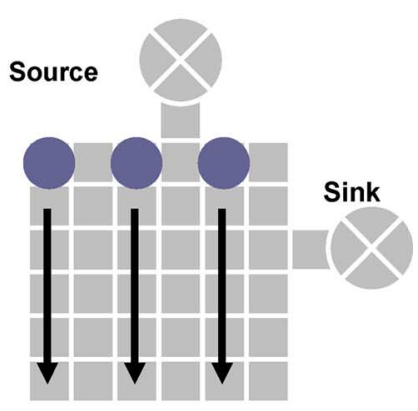

(d)

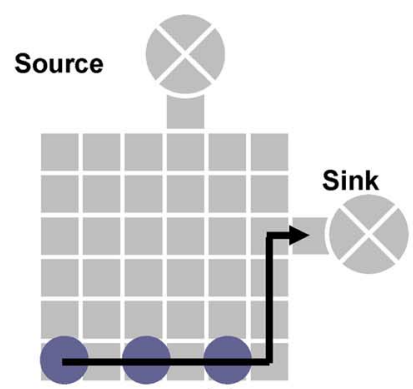

(e)

Fig. 7. Step II. (a) Parallel scan-like test for even columns. (b) Routing of test droplets to sink. (c) Test droplet routed to odd columns. (d) Parallel scan-like test for odd columns. (e) Routing of test droplets to sink.

every single cell and all "edges" (pairs of adjacent cells) in each column. Therefore, it is referred to as "column test."

Step III. Row Test: Repeat parallel scan-like test (two iterations) for the rows to detect defects involving pairs of adjacent cells in each row. This step is referred to as "row test."

We next use a $6 \times 6$ array as an example. A total of three test droplets $(6 / 2=3)$ are dispensed and routed in each parallel scan-like test iteration. The various steps in the test application procedure are shown in Figs. 6-8. 


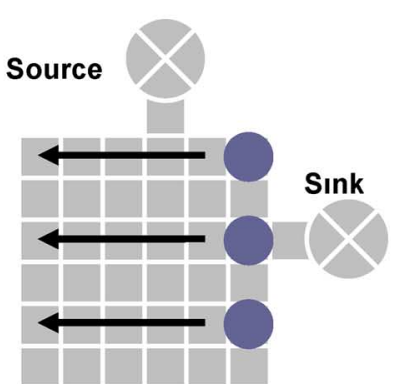

(a)

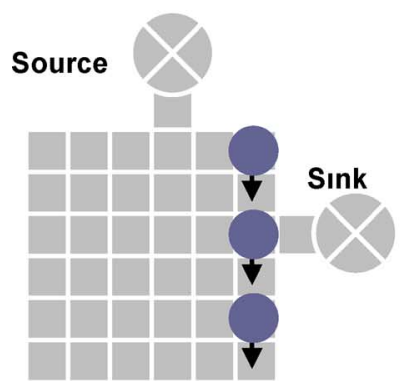

(c)

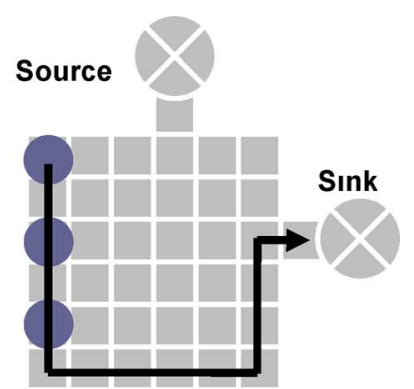

(b)

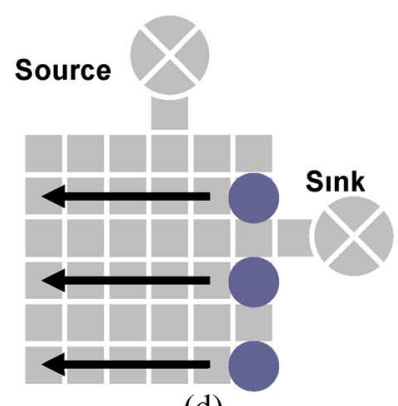

(d)

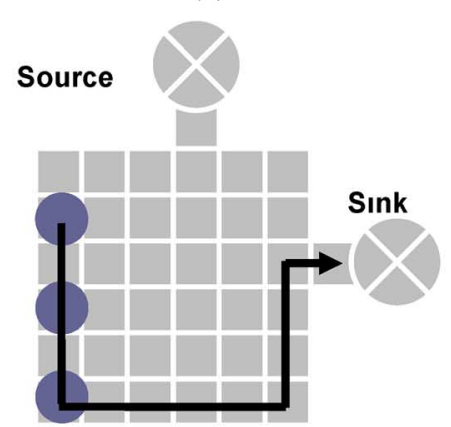

(e)

Fig. 8. Step III. (a) Parallel scan-like test for odd rows. (b) Routing of test droplets to sink. (c) Test droplet routed to even rows. (d) Parallel scan-like test for even rows. (e) Routing of test droplets to sink.

In order to achieve defect tolerance via reconfiguration, a diagnosis method is needed to locate faulty cells. We do not attempt to identify the defect type in this work. We only distinguish between a defect involving one cell and a defect involving two cells, i.e., an electrode-short. We next present an efficient diagnosis procedure based on parallel scan-like testing.

For defect-free chips, test droplets, which start simultaneously at the corresponding pseudo-sources, traverse their target columns/rows and reach their pseudo-sinks at the same time. The droplets are then routed from the pseudo-sinks to the sink, trigging a pulse sequence, as shown in Fig. 5(b). If there is a defect in a row or a column, the corresponding droplet will not arrive at the pseudo-sink. Different pulse patterns correspond to different defect locations. Consider the example shown in Fig. 9. The output pulse sequence indicates a defect in the fifth column. The defect site can be precisely identified by carrying out the row tests. In some cases, it is difficult to map test outcomes to candidate fault patterns, e.g., a test outcome missing the first pulse is the same as that missing the last pulse. To solve this problem, the arrival time for the first test droplet is calculated before test application; in this way, we get a reference point in order to avoid ambiguous interpretation of test outcomes.

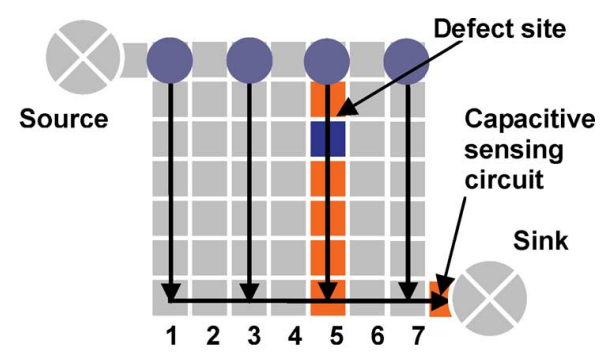

Test

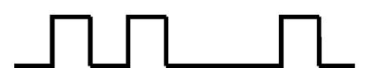

Fig. 9. Example of test outcome for a faulty array.

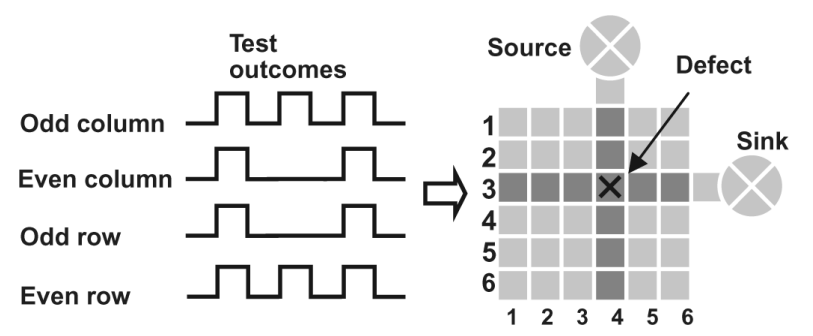

Fig. 10. Diagnosis of a single-electrode defect by "cross-parallel" scan-like test.

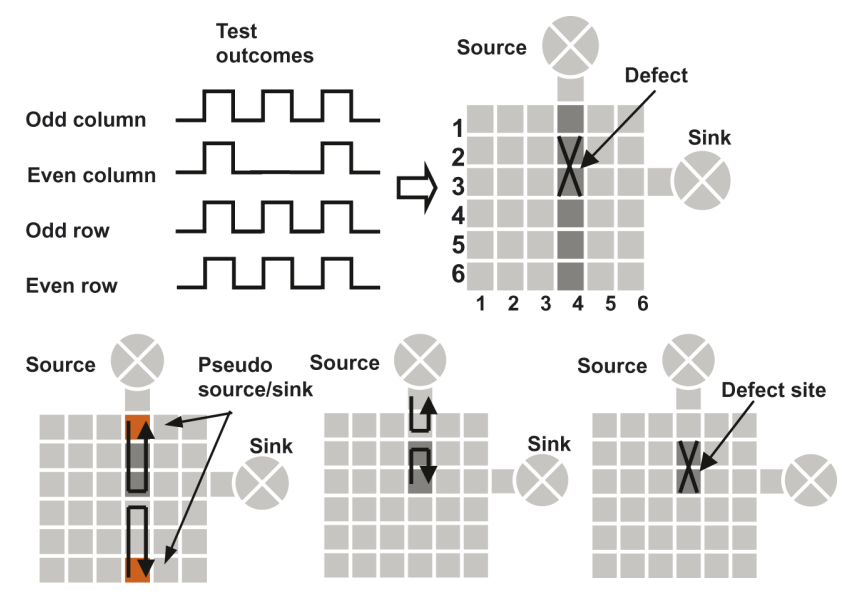

Fig. 11. Diagnosis of an electrode-short defect using parallel scan-like test.

Using the above method, a single faulty cell can be located as shown in Fig. 10; the test droplet will be stuck at the faulty cell in both the column-test and row-test steps. If the defect is an electrode-short, the test droplet will be stuck at the short site in either the row-test or column-test, but not both. No additional diagnosis steps are needed. In contrast, Euler-path-based testing [19] reply on a binary search process to determine the exact location of the defect. Thus, parallel scan-like test saves time for many testing applications where only defect-type information is needed.

Defect localization is more complicated when the fault is due to a short between two electrodes. In this case, the sensor readout indicates an error for only one step, i.e., either columntest or row-test; see Fig. 11. A binary-search method can next be used to locate the shorted electrode pair by iteratively partitioning the column/row and carry out single line parallel scanlike test, as shown in Fig. 11. Compared to the Euler-circuit 


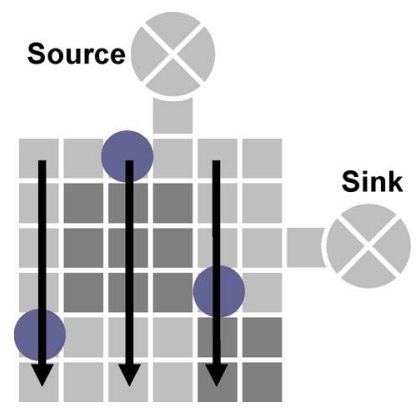

Fig. 12. Online parallel scan-like test for a $6 \times 6$ microfluidic array. Shaded cells correspond to modules in use for bioassay operations.

based method, the two-dimensional array partitioning of [19] is simplified to 1-D. As a result both number of test iterations and the complexity of each iteration are reduced, which leads to a significant decrease in diagnosis time.

The above discussion on diagnosis assumes a single faulty cell. The procedure can also be used to locate multiple faulty cells, but it does not guarantee that only faulty cells are placed in the set of candidate defect sites.

\section{B. Online Parallel Scan-Like Test}

The proposed parallel scan-like test method can also be used for online testing with a few modifications. First, note that some test droplets are stalled while the target cells are occupied by the sample and reagent droplets needed for the bioassay. Therefore, some test droplets may be out of step with each other, as shown in Fig. 12. The three droplets arrive at the pseudo-sinks at different times. Second, test droplets are routed to the sink only after all the test droplets arrive at the boundary. While this procedure leads to an increase in testing time, it guarantees a regular output at the detection sensor that is easy to read, therefore the capacitive sensing circuit can be kept simple. Online fault diagnosis, although based on the same idea as offline diagnosis, is more complicated since test droplets are moving out-of-step with each other. To determine the arrival time for droplet detection, operation scheduling and module placement results [22] are used to calculate the waiting time for test droplets. Once arrival times are determined, online fault diagnosis can be carried out using the same procedure presented earlier for offline diagnosis.

\section{Diagnosis of Multiple Defects}

The proposed parallel scan-test method efficiently tests the target biochip and locates defects. However, it is not able to always unambiguously and accurately locate multiple defect sites. In this section, we integrate a redundant test method into the parallel scan-like test technique to address this problem.

\section{A. Incorrectly Classified Defects}

When multiple defects exist in the array, multiple columns and multiple rows might fail during the parallel scan-like test method.

However, unlike in the case of a single defect, we cannot identify the multiple defect locations by simply examining the failing columns and rows. This is because the failing columns

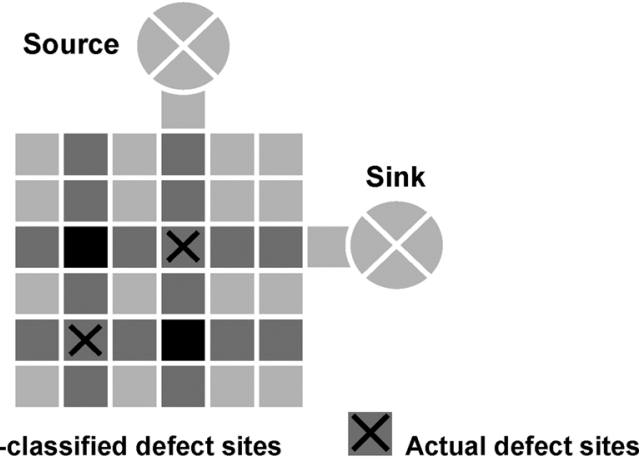

Fig. 13. Example of incorrectly classified defects.

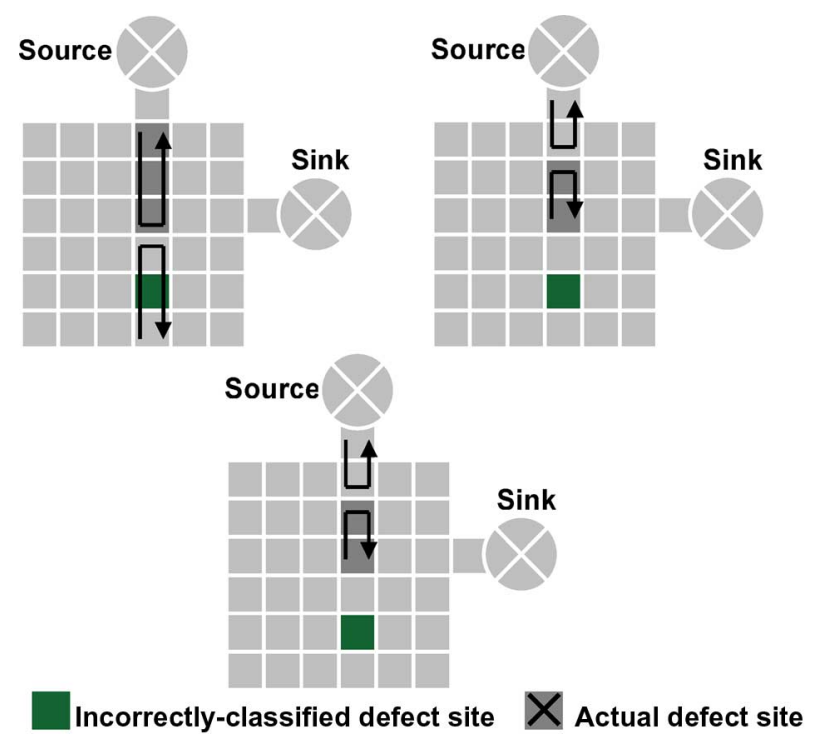

Fig. 14. Elimination of incorrectly classified defects using binary-search-based diagnosis.

and rows intersect not only at the defect site but also at some defect-free electrodes, which are referred to as incorrectly classified defect sites. This problem is illustrated in Fig. 13.

The above problem can be solved by carrying out binary search for each column/row that fails the test, as shown in Fig. 14. This method eliminates the likelihood of incorrectly classified defects false and helps us to precisely locate the actual defect sites. However, it suffers from the drawback that precise defect localization is not possible when there are "untestable sites" in the array, a problem described next.

\section{B. Untestable Sites}

An untestable site is defined as an electrode that cannot be traversed by any test droplets in the parallel scan-like test method. A site becomes untestable when there are defects in both its row and column, and in all four directions, i.e., North, South, East, and West, as shown in Fig. 15. In this case, test droplets are impeded by the defects and they cannot reach the untestable site.

The above problem can be addressed by carrying out one more iteration of parallel scan-like test. As in column and row test, we referred to this test iteration as diagonal test, as shown in Fig. 16. In the additional test iteration, multiple test droplets are manipulated to traverse the array from one diagonal direction 


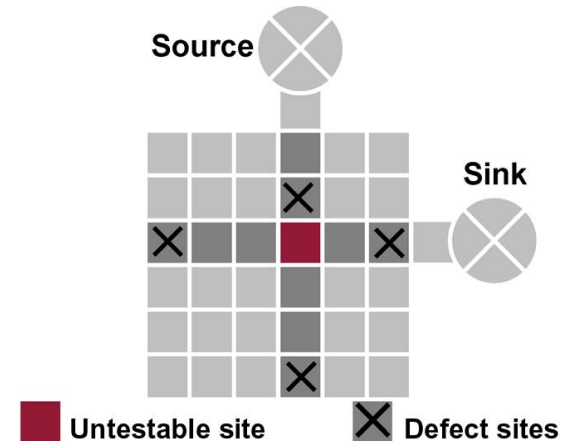

Fig. 15. Example of an untestable site.

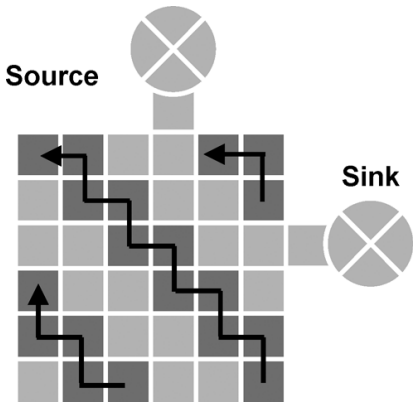

Fig. 16. Illustration of diagonal parallel scan-like test.

(top-left to bottom-right, or top-right to bottom-left) or both, referred to here as single-diagonal test and cross-diagonal test, respectively. Untestable sites that cannot be reached from vertical and horizontal directions can be reached diagonally. Even though this approach cannot guarantee the testability of all the untestable sites, it significantly reduces the probability that an electrode site is untestable.

Another advantage of the diagonal test procedure is that it can also help in the avoidance of incorrectly classified defect sites. Only the sites that lie on failing columns, failing rows, and failing diagonals are identified as defects. Although this method does not completely eliminate the likelihood of incorrectly classified defects, it provides acceptable diagnostic resolution by eliminating most incorrectly classified defects. These advantages are highlighted quantitatively in Section VI-B.

\section{PeRformance Evaluation}

In this section, we evaluate the performance of the proposed testing and diagnosis method. We first carry out complexity analysis of parallel scan-like testing and compare it with results obtained from the Euler-path-based method [19]. Next, we use probabilistic analysis to evaluate the improvement in diagnostic resolution obtained using the proposed technique for locating multiple defects.

\section{A. Complexity Analysis}

We first calculate the complexity of parallel scan-like testing. For simplicity we only discuss offline test of a target array that contains a single defect. The parallel scan-like test method is based on three stages, i.e., peripheral test, column-test and row-test.

To test a $N \times N$ target array, peripheral test is first carried out, and this stage takes which take $4 N$ steps. Each step is defined as a droplet manipulation from one electrode to another, which takes 1 second for a typical actuation frequency of $1 \mathrm{~Hz}$. Next, column- and row-tests are carried out, each takes $N$ steps. Thus, the total test procedure includes $8 N$ steps, i.e., $O(N)$. Fault diagnosis is based on one-dimensional binary partitioning, therefore it is also $O(N)$. Compared to Euler-path-based method, which has $O\left(N^{2}\right)$ complexity, the time needed for both testing and diagnosis are significantly reduced.

To make a more practical comparison, we apply the proposed parallel scan-like test method and the Euler-path-based method to the offline testing of microfluidic arrays with sizes varying from $10 \times 10$ to $50 \times 50$ electrodes. Note that the complexity for both the proposed method and the Euler-path-based method is independent of defect location. Thus, for each size, a sample array with a randomly injected faulty cell is generated as a target array. To get the precise test time for the proposed method, we calculate the time needed to route the droplet from source to the pseudo-source and from pseudo-sink to the sink reservoir, and add to the test time derived using the above complexity analysis. The results are shown in Fig. 17.

As predicted by the complexity analysis, the test time for the Euler-path-based testing increases quadratically with the array size, while the parallel scan-like test time increases only linearly. A significant improvement can be seen for large arrays.

\section{B. Probabilistic Analysis}

Next we calculate the probability of the occurrence of incorrectly classified defects, i.e., the probability of an electrode to be a candidate defect site when it is not defective. Assume that each electrode fails independently with probability $p$. The probability that an electrode is defect-free is therefore simply $1-p$. An electrode is a candidate defective electrode if there is an actual defect in either the same column or the same row as this electrode; see Fig. 18. Therefore, the probability $P_{\text {cand }}$ that a defect-free electrode is classified as a candidate defect site in an $N \times N$ array is given by

$$
P_{\text {cand }}=(1-p)\left(1-(1-p)^{N-1}\right)^{2}
$$

For $p \ll 1$, and large $N$, we get

$$
\begin{aligned}
P_{\text {cand }} & =(1-p)\left(1-(1-p)^{N-1}\right)^{2} \\
& \approx p^{2}(1-p)(N-1)^{2} \\
& =p^{2}(1-p) N^{2}
\end{aligned}
$$

When diagonal testing is carried out, a defect-free electrode is classified as a candidate electrode only if the following conditions hold: 1) there is a defect in the same column as the electrode; 2) there is a defect in the same row as the electrode; and 3 ) there are a defect on one or both the diagonals on which the electrode lies. These situations are illustrated in Fig. 19(a) and Fig. 19(b). 


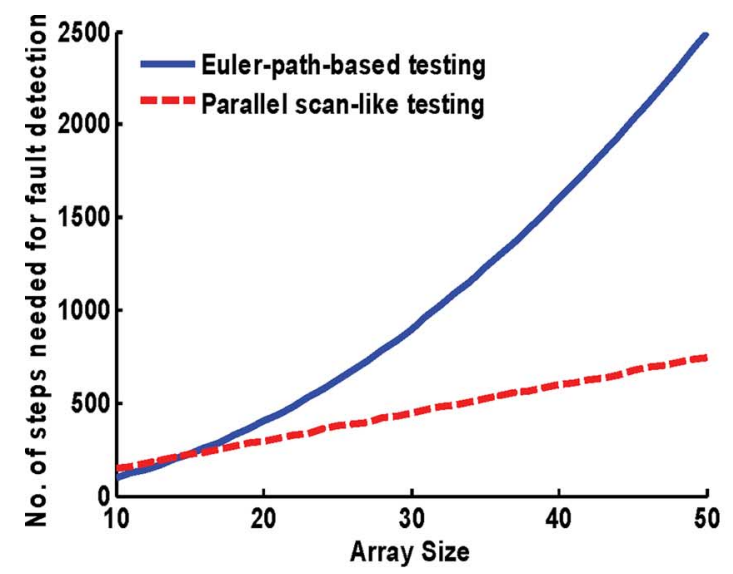

(a)

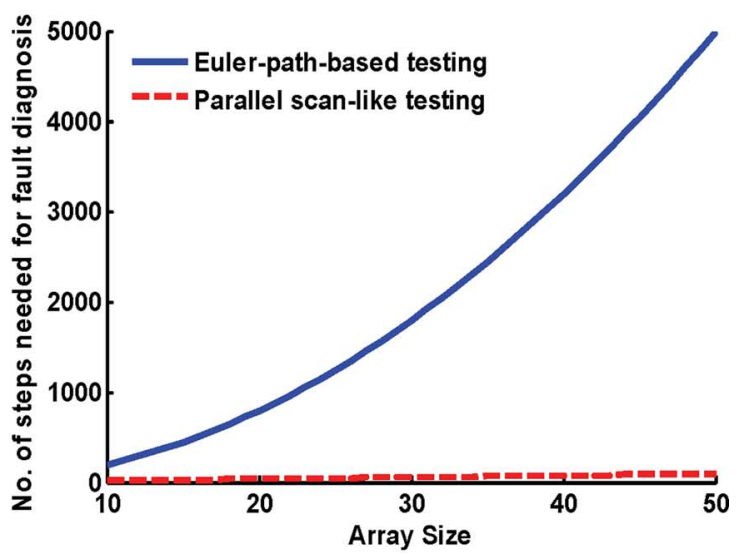

(b)

Fig. 17. Comparison of (a) testing complexity, and (b) diagnosis complexity (additional steps) of parallel scan-like test and Euler-circuit based method.

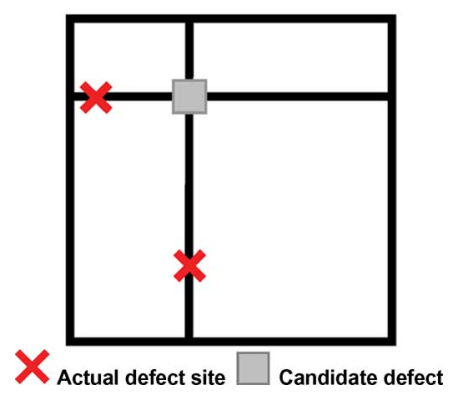

Fig. 18. Illustration of a candidate defect (incorrectly classified).

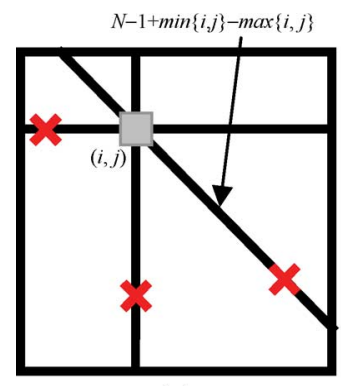

(a)

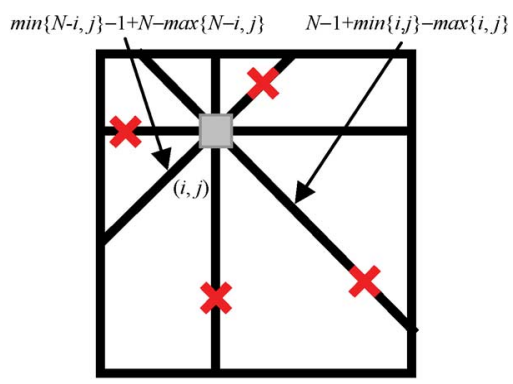

(b)
Fig. 19. Illustration of the conditions that lead to incorrect classification of candidate defects for (a) single-diagonal diagnosis, (b) cross-diagonal diagnosis.

If single-diagonal diagnosis is carried out as in Fig. 19(a), the probability $P_{\text {incorrect }}$ of an incorrect classification for an electrode is given by

$$
\begin{aligned}
P_{\text {incorrect }}=(1-p)( & \left.-(1-p)^{N-1}\right)^{2} \\
& \times\left(1-(1-p)^{N-1+\min \{i, j\}-\max \{i, j\}}\right)
\end{aligned}
$$

If cross diagonal diagnosis is carried out as in Fig. 19(b), the probability of false defect is

$$
\begin{array}{r}
(1-p)\left(1-(1-p)^{N-1}\right)^{2}\left(1-(1-p)^{N-1+\min \{i, j\}-\max \{i, j\}}\right) \\
\times\left(1-(1-p)^{N-1+\min \{N-i, j\}-\max \{N-i, j\}}\right) .
\end{array}
$$

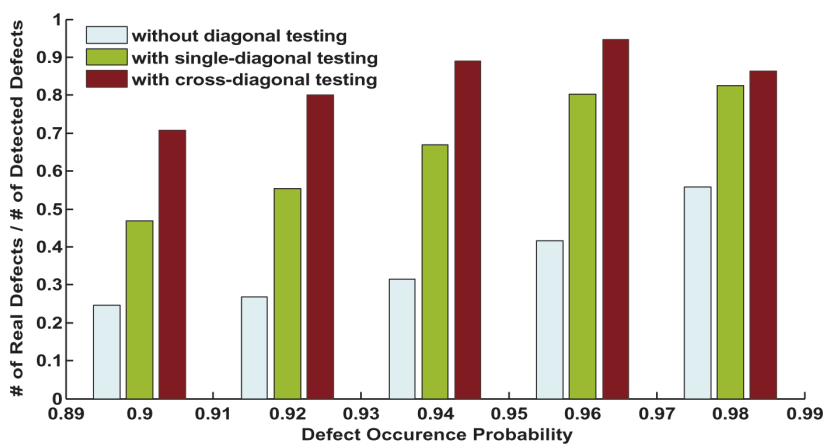

Fig. 20. Simulation results highlighting the likelihood of incorrect classification.

Using these equations, we calculate the probability of false defect occurrence under different probability of defect occurrence. The results are shown in Fig. 20.

In Fig. 20, it can be seen that a significant increase in diagnostic resolution, i.e., the ratio of the number of actual defects to the number of classified defects, is achieved by carrying out single-diagonal diagnosis. Further improvement can be achieved when cross-diagonal diagnosis is applied. However, the difference in the results for cross-diagonal diagnosis and single-diagonal diagnosis is less apparent for smaller values of the defect occurrence probability.

1) Occurrence Probability of Untestable Sites: Next we analyze the probability of the occurrence of untestable sites. Again assume each electrode is failing with the same probability of $p$. The electrode is untestable if there is one real defect in each of its four directions, as shown in Fig. 15. Therefore, for a single electrode in row $i$ and column $j$, the probability $P_{\text {untest }}$ that it cannot be tested is

$$
\begin{aligned}
P_{\text {untest }}=\left(1-(1-p)^{i-1}\right)\left(1-(1-p)^{N-i}\right) & \\
& \times\left(1-(1-p)^{j-1}\right)\left(1-(1-p)^{N-j}\right) .
\end{aligned}
$$

When diagonal testing is carried out, the electrode is untestable only if there is also a real defect in the same diagonal or diagonals as shown in Fig. 21(a) and (b). 


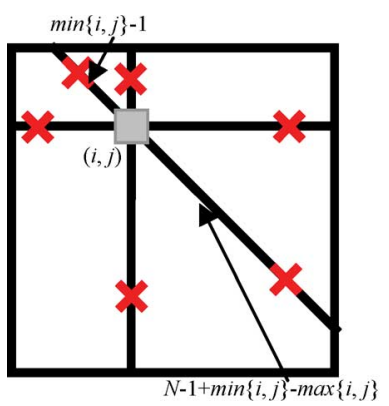

(a)

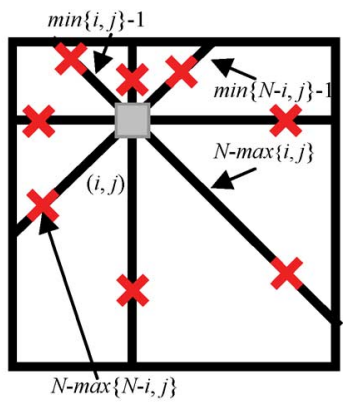

(b)
Fig. 21. Illustration of untestable sites for (a) single-diagonal diagnosis (b) cross-diagonal diagnosis.

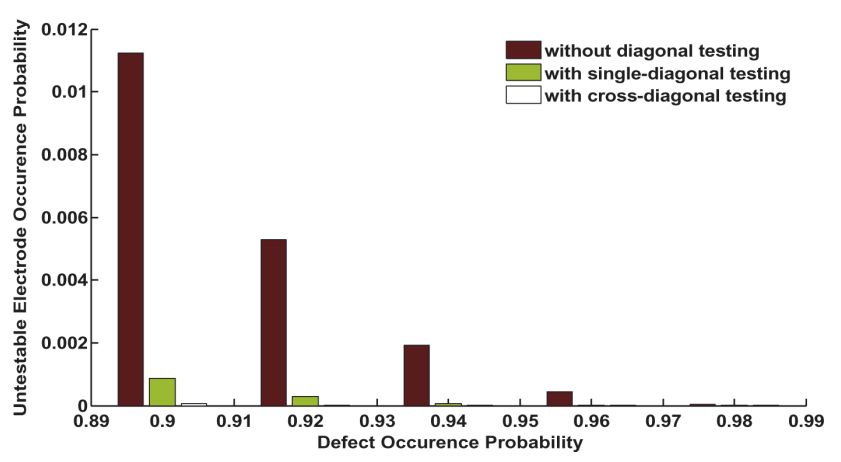

Fig. 22. Simulation results highlighting the probability of untestable sites.

If single-diagonal diagnosis is carried out as in Fig. 21(a), the probability $P_{\text {untest }}$ of an untestable electrode is

$$
\begin{aligned}
P_{\text {untest }}= & \left(1-(1-p)^{i-1}\right)\left(1-(1-p)^{N-i}\right)\left(1-(1-p)^{j-1}\right) \\
& \cdot\left(1-(1-p)^{N-j}\right)\left(1-(1-p)^{\min \{i, j\}-1}\right) \\
& \cdot\left(1-(1-p)^{N-\max \{i, j\}}\right) .
\end{aligned}
$$

If cross-diagonal diagnosis is carried out as in Fig. 21(b), the probability $P_{\text {untest }}$ of an untestable electrode is given by

$$
\begin{aligned}
P_{\text {untest }}= & \left(1-(1-p)^{i-1}\right)\left(1-(1-p)^{N-i}\right)\left(1-(1-p)^{j-1}\right) \\
& \cdot\left(1-(1-p)^{N-j}\right)\left(1-(1-p)^{\min \{i, j\}-1}\right) \\
& \times\left(1-(1-p)^{N-\max \{i, j\}}\right)\left(1-(1-p)^{\min \{N-i, j\}-1}\right) \\
& \times\left(1-(1-p)^{N+\max \{N-i, j\}}\right) .
\end{aligned}
$$

Using the above formulas, we calculate the probability of false defect occurrence under different probability of untestable electrode occurrence. The results are shown in Fig. 22. We see that diagonal testing leads to a significant reduction in the probability that a cell is untestable. Even though the proposed multiple-defect diagnosis method does not guarantee the testability of all electrodes, it reduce the occurrence probability of untestable sites to almost zero (less than 0.0001).

\section{APPLICATION tO A FABRICATED BIOCHIP}

In this section, we apply the parallel scan-like test method to a fabricated biochip. The chip-under-test is a PCB microfluidic platform for DNA sequencing, as shown in Fig. 23. The platform consists of a $7 \times 7$ array, 8 reservoirs and routing electrodes that

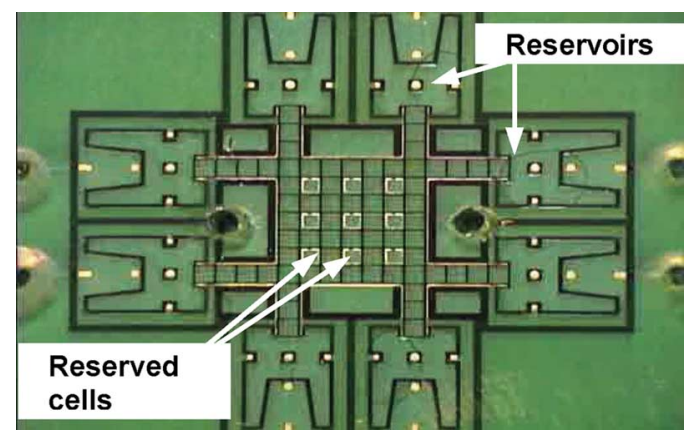

Fig. 23. Fabricated biochip for DNA sequencing.
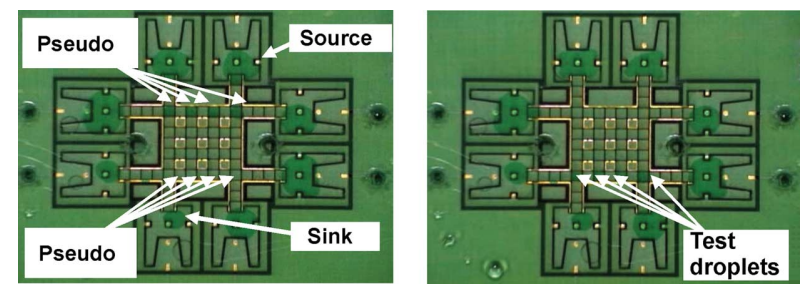

Fig. 24. Column-test step of parallel scan-like test.

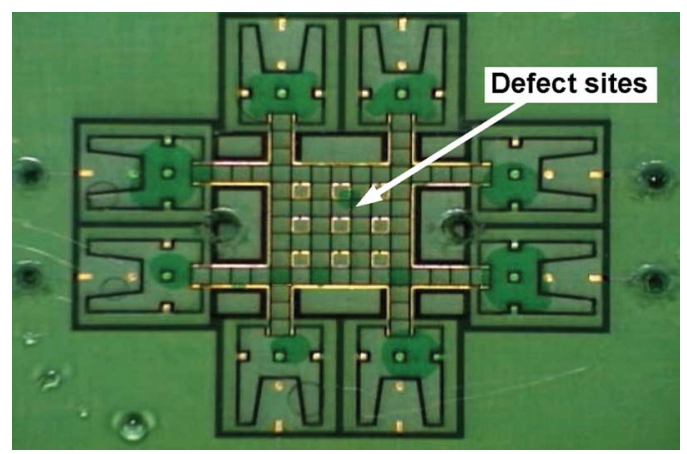

Fig. 25. Parallel scan-like diagnosis of single cell defect.

connect reservoirs to the array. A total of 9 cells are reserved for grounding, and they are not available for droplet transportation.

As a baseline, we first apply Euler-path-based testing to this chip. The test procedure takes $57 \mathrm{~s}$, assuming a (typical) $1-\mathrm{Hz}$ electrode-actuation frequency. Next we carry out parallel scanlike test (the column-test stage is shown in Fig. 24). Since 9 electrodes are not reachable, the testing of even columns and rows are not need. The test application procedure takes $46 \mathrm{~s}$, again for a $1-\mathrm{Hz}$ actuation frequency.

Next we study the time needed for fault diagnosis for the two methods. We use a fabricated chip, which is known to contain one defect a priori (determined by inspection and electrical measurements). The chip with the defect is shown in Fig. 25. For the Euler-path-based method, we carried out a binary search to locate the defect cell. A total of seven iterations are needed and the total diagnosis time is $173 \mathrm{~s}$. This value is obtained by summing up the times for the different diagnosis iterations which are 57, 44, 32, 16, 8, 4, and $2 \mathrm{~s}$, respectively. On the other hand, parallel scan-like test can simply determine the defect site from testing readouts. No additional diagnosis steps are needed and the diagnosis time is the same as the testing time, i.e., $44 \mathrm{~s}$, which correspond to a $75 \%$ reduction compared to [19]. 


\section{CONCLUSION}

We have proposed an efficient test and diagnosis method for digital microfluidic biochips. The proposed method enables parallel testing using multiple test droplets for both online and offline testing. We have also identified a number of common defects and defect types. These causes of defects have been related to fault models and observable errors. The proposed test and diagnosis method have been evaluated using complexity analysis and a fabricated chip example. The results obtained demonstrate significant improvement over prior work on the testing and diagnosis of digital microfluidic biochips.

\section{REFERENCES}

[1] R. B. Fair et al., "Electrowetting-based on-chip sample processing for integrated microfluidics," in Proc. IEDM, 2003, pp. 32.5.1-32.5.4.

[2] E. Verpoorte and N. F. De Rooij, "Microfluidics meets MEMS," Proc. IEEE, vol. 91, no. 6, pp. 930-953, Jun. 2003.

[3] J. Zeng and T. Korsmeyer, "Principles of droplet electrohydrodynamics for lab-on-a-chip," Lab on a Chip, vol. 4, pp. 265-277, 2004.

[4] D. Chatterjee et al., "Droplet-based microfluidics with nonaqueous solvents and solutions," Lab on a Chip, vol. 6, pp. 199-206, 2006.

[5] S. K. Cho et al., "Creating, transporting, cutting, and merging liquid droplets by electrowetting-based actuation for digital microfluidic circuits," J. Microelectromech. Syst., vol. 12, pp. 70-80, 2003.

[6] P. S. Dittrich et al., "Micro total analysis systems: Latest advancements and trends," Anal. Chem., vol. 78, pp. 3887-3908, 2006.

[7] R. B. M. Schasfoort et al., "Field-effect flow control for microfabricated fluidic networks," Science, vol. 286, pp. 942-945, 1999.

[8] B. M. Paegel et al., "Microfluidic devices for DNA sequencing: Sample preparation and electrophoretic analysis," Current Opin. Biotechnol., vol. 14 , pp. 42-50, 2003.

[9] "Two-dimensional digital microfluidic system by multi-layer printed circuit board," in Proc. IEEE MEMS, 2005, pp. 726-729.

[10] T. Xu and K. Chakrabarty, "Parallel scan-like testing and fault diagnosis techniques for digital microfluidic biochips," in Proc. IEEE Eur. Test Symp., 2007, pp. 63-68.

[11] H. G. Kerkhoff, "Testing of microelectronic-biofluidic systems," IEEE Des. Test. Comput., vol. 24, no. 1, pp. 72-82, Jan./Feb. 2007.

[12] H. G. Kerkhoff and M. Acar, "Testable design and testing of micro-electro-fluidic arrays," in Proc. IEEE VLSI Test Symp., 2003, pp. 403-409.

[13] H. G. Kerkhoff and H. P. A. Hendriks, "Fault modeling and fault simulation in mixed micro-fluidic microelectronic systems," in JETTA, 2001, vol. 17 , pp. $427-437$.

[14] F. Su et al., "Testing of droplet-based microelectrofluidic systems," in Proc. IEEE Int. Test Conf., 2003, pp. 1192-1200.

[15] F. Su et al., "Ensuring the operational health of droplet-based microelectrofluidic biosensor systems," IEEE J. Sens., vol. 5, no. 4, pp. 763-773, Aug. 2005.

[16] F. Su et al., "Concurrent testing of droplet-based microfluidic systems for multiplexed biomedical assays," in Proc. Int. Test Conf., 2004, pp. 883-892.

[17] T. H. Schulte et al., "Microfluidic technologies in clinical diagnostics," Clinica Chim. Acta, vol. 321, pp. 1-10, 2002.

[18] A. Itai et al., "Hamilton paths in grid graphs," SIAM J. Comput., vol. 11, pp. 676-686, 1982.

[19] F. Su et al., "Defect-oriented testing and diagnosis of digital microfluidics-based biochips," in Proc. IEEE Int. Test Conf., 2005, pp. 487-496.

[20] Silicon Biosystems [Online]. Available: http://www.siliconbiosystems. com/applications/webwork/DEPArray.page

[21] F. Su et al., "Droplet routing in the synthesis of digital microfluidic biochips," in Proc. DATE Conf., 2006, pp. 323-328.
[22] F. Su and K. Chakrabarty, "Unified high-level synthesis and module placement for defect-tolerant microfluidic biochips," in Proc. IEEE/ACM Des. Automat. Conf., 2005, pp. 825-830.

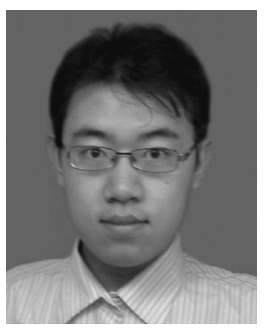

Tao Xu (S'07) received the B.E. degree in electrical engineering from Zhejiang University, Hangzhou, China, in 2005 and the M.S. degree in electrical and computer engineering from Duke University, Durham, NC, in 2007, where he is currently pursuing the Ph.D. degree in electrical and computer engineering.

His research interests include design and testing of mixed-technology microsystems, electronic design automation, mixed-signal VLSI design, MEMS modeling and simulation.

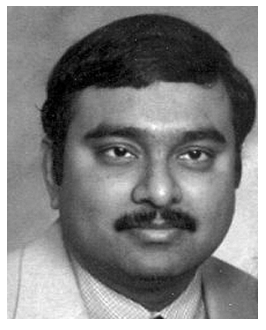

Krishnendu Chakrabarty (S'92-M'96-SM'00) received the $\mathrm{B}$.Tech. degree in computer science and engineering from the Indian Institute of Technology, Kharagpur, India in 1990, and the M.S.E. and Ph.D. degrees in computer science and engineering from the University of Michigan, Ann Arbor, in 1992 and 1995 , respectively, all in computer science and engineering.

$\mathrm{He}$ is currently a Professor of electrical and computer engineering at Duke University, Durham, NC. His current research projects include: testing and testing and design-for-testability of system-on-chip integrated circuits; digital microfluidic biochips; logic circuits based on DNA self-assembly; delay-tolerant wireless networks. He has authored five books: Microelectrofluidic Systems: Modeling and Simulation (CRC Press, 2002); Test Resource Partitioning for System-on-a-Chip (Kluwer, 2002); Scalable Infrastructure for Distributed Sensor Networks (Springer, 2005); Digital Microfluidics Biochips: Synthesis, Testing, and Reconfigutaion Techniques (CRC Press, 2006); and Adaptive Cooling of Integrated Circuits using Digital Microfluidics (Artech House, April 2007) and edited the book volumes SOC (System-on-a-Chip) Testing for Plug and Play Test Automation (Kluwer, 2002) and Design Automation Methods and Tools for Microfluidics-Based Biochips (Springer, 2006). He has contributed over a dozen invited chapters to book volumes, published 250 papers in archival journals and refereed conference proceedings, and delivered over 100 keynote, plenary, and invited talks. He holds a U.S. patent in built-in self-test and is a co-inventor of a pending U.S. patent on sensor networks.

Prof. Chakrabarty is a recipient of the National Science Foundation Early Faculty (CAREER) award, the Office of Naval Research Young Investigator award, and Best Paper awards at the 2007 IEEE International Conference on VLSI Design, the 2005 IEEE International Conference on Computer Design, and the 2001 IEEE Design, Automation and Test in Europe (DATE) Conference. He is also a recipient of the Humboldt Research Fellowship, awarded by the Alexander von Humboldt Foundation, Germany, and the Mercator Visiting Professorship, awarded by the Deutsche Forschungsgemeinschaft, Germany. He is a Distinguished Visitor of the IEEE Computer Society for 2005-2007 and a Distinguished Lecturer of the IEEE Circuits and Systems Society for 2006-2007. He is an Associate Editor of IEEE TRANSACTIONS ON COMPUTER-AIDED DESIGN OF INTEGRATED CiRCUITS AND SYSTEMS, IEEE TRANSACTIONS ON VERY LaRge SCALE InTEGRATEd Systems, IEEE TRANSACtions on Circuits and Systems-I: REgular PaPers, IEEE TRANSACTIONS ON BIOMEDICAL CIRCUITS AND SYSTEMS, ACM Journal on Emerging Technologies in Computing Systems, an Editor of IEEE Design and Test of Computers, and an Editor of Journal of Electronic Testing: Theory and Applications (JETTA). He served as Program Chair for the IEEE Asian Test Symposium in 2005 and the CAD, Design, and Test Conference for the 2007 IEEE Symposium on Design, Integration, Test, and Packaging of MEMS/MOEMS. He is a senior member of ACM and a member of Sigma Xi. 